\title{
The metallicity of the most distant quasars ${ }^{\star}$
}

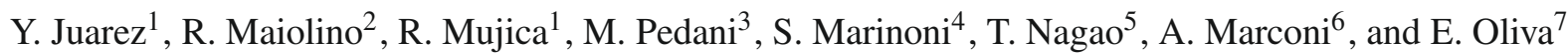 \\ 1 Instituto Nacional de Astrofísica, Óptica y Electrónica, Puebla, Luis Enrique Erro 1, Tonantzintla, Puebla 72840, Mexico \\ 2 INAF - Osservatorio Astronomico di Roma, via di Frascati 33, 00040 Monte Porzio Catone, Italy \\ e-mail: maiolino@oa-roma.inaf.it \\ 3 Large Binocular Telescope Observatory, University of Arizona, 933 N. Cherry Ave., Tucson, AZ 85721-0065, USA \\ ${ }^{4}$ INAF - Telescopio Nazionale Galileo, PO Box 565, 38700 Santa Cruz de La Palma, Tenerife, Spain \\ 5 Research Center for Space and Cosmic Evolution, Ehime University, 2-5 Bunkyo-cho, Matsuyama 790-8577, Japan \\ ${ }^{6}$ Dipartimento di Astronomia, Università di Firenze, Largo E. Fermi 2, 50125 Firenze, Italy \\ 7 Osservatorio Astrofisico di Arcetri, Largo E. Fermi 5, 50125 Firenze, Italy \\ Received 25 November 2008 / Accepted 29 December 2008
}

\section{ABSTRACT}

\begin{abstract}
We investigate the metallicity of the broad line region (BLR) of a sample of 30 quasars in the redshift range $4<z<6.4$, by using near-IR and optical spectra. We focus on the ratio of the broad lines (SiIV1397+OIV]1402)/CIV1549, which is a good metallicity tracer of the BLR. We find that the metallicity of the BLR is very high even in QSOs at $z \sim 6$. The inferred metallicity of the BLR gas is so high (several times solar) that metal ejection or mixing with lower metallicity gas in the host galaxy is required to match the metallicities observed in local massive galaxies. On average, the observed metallicity changes neither among quasars in the observed redshift range $4<z<6.4$, nor when compared with quasars at lower redshifts. We show that the apparent lack of metallicity evolution is a likely consequence of both the black hole-galaxy co-evolution and of selection effects. The data also suggest a lack of evolution in the carbon abundance, even among $z>6$ quasars. The latter result is puzzling, since the minimum enrichment timescale of carbon is about $1 \mathrm{Gyr}$, i.e. longer than the age of the universe at $z \sim 6$.
\end{abstract}

Key words. ISM: abundances - galaxies: abundances - galaxies: evolution - galaxies: high-redshift galaxies: quasars: emission lines

\section{Introduction}

One of the important results from studies of high-redshift quasars is the apparent lack of evolution in their emission properties. In particular, the lack of evolution in the emission-line ratios is interpreted as a lack of metallicity evolution among QSOs at different redshifts. Previous tentative indications of metallicity evolution with redshift were later ascribed to a metallicity dependence on the quasar luminosity (Nagao et al. 2006a), which may mimic a dependence on redshift in flux-limited samples. Warner et al. (2004) and Shemmer et al. (2004) suggest that the metallicity-luminosity relation may actually result from a $\mathrm{BH}-$ metallicity dependence or from a relation between accretion rate $\left(L / L_{\text {Edd }}\right)$ and metallicity.

At $z>5$ most of the emission lines used to constrain the BLR metallicity are redshifted into the near-IR. As a consequence the investigation of the BLR metallicity at $z>5$ has been limited to a small number of objects. Various authors have investigated the iron abundance in $z \sim 6$ QSOs by using near-IR spectra to measure the intensity of the UV "FeII-bump" $\left(\lambda_{\text {rest }} \sim 2000\right.$ $3050 \AA$ ) relative to the MgII $\lambda 2789$ line (Freudling et al. 2003; Maiolino et al. 2003; Iwamuro et al. 2004; Kurk et al. 2007), as a proxy of the $\mathrm{Fe} / \alpha$-elements abundance ratio. No evolution was found in the ratio FeII/MgII; however, since the FeII bump is a strong BLR coolant, its strength depends on several other parameters and may not depend primarily on the iron abundance.

^ Based on data obtained at the VLT, through the ESO programs 074.B-0185 and 075.B-0487, and at the Telescopio Nazionale Galileo.
Pentericci et al. (2002) investigated the metallicity in the BLR of two QSOs at $z \sim 6$ by measuring the ratio of the CIV1549 and NV1240 emission lines, suggesting super-solar metallicities. However, the calibration of the ratio NV/CIV as a tracer of gas metallicity is mostly based on the assumption that nitrogen is a secondary element and that, therefore, its abundance scales quadratically with metallicity, i.e. $\mathrm{N} / \mathrm{H} \propto(\mathrm{O} / \mathrm{H})^{2}$. The latter assumption may, however, be an oversimplification and may give unrealistically high metallicities, since the nitrogen abundance actually depends on the detailed star formation history (Bresolin et al. 2004; Jiang et al. 2008).

Nagao et al. (2006a) show that the ratio (SiIV $\lambda 1397+$ OIV] $\lambda 1402$ )/CIV $\lambda 1549$ (hereafter (SiIV+OIV)/CIV) is a tracer of the BLR metallicity, the reason mainly being that the relative importance of CIV as a coolant decreases with the BLR metallicity Ferland et al. (1996). This method has the advantage of not relying on the assumption $\mathrm{N} / \mathrm{H} \propto(\mathrm{O} / \mathrm{H})^{2}$. Moreover, (SiIV+OIV) and CIV are well isolated features, which do not require difficult deblending techniques and which can be measured even in low-resolution spectra. Jiang et al. (2007) used near-IR spectra to measure (SiIV+OIV)/CIV in five QSOs at $z \sim 6$, and find no evidence of evolution when compared with lower redshift QSOs.

In this paper we present measurements of the (SiIV + OIV)/CIV ratio in 30 QSOs at $4<z<6.4$ obtained by means of near-IR spectra, with the goal of better investigating the metallicity evolution of QSOs in the early Universe. In the following we adopt the following cosmological parameters: $H_{0}=70 \mathrm{~km} \mathrm{~s}^{-1} \mathrm{Mpc}^{-1}, \Omega_{\mathrm{M}}=0.3$, and $\Omega_{\Lambda}=0.7$. 


\section{Observations}

We observed a sample of 30 high-redshift quasars $(4.0<$ $z<6.4)$ from the SDSS by means of near-IR and optical spectra covering at least the UV rest-frame emission lines SiIV $\lambda 1397+O I V] \lambda 1402$ and CIV $\lambda 1549$, but in most cases the spectra extend to $\lambda_{\text {rest }} \sim 3000-4000 \AA$. The original goal of most of the observations was to constrain the dust extinction in high- $z$ QSOs. A more detailed description of the data and the results on the dust extinction will be given in Gallerani et al. (in prep.). Here we only focus on a byproduct, namely the evolution of the BLR metallicity based on the (SiIV+OIV)/CIV ratio.

Observations were obtained both with the Italian telescopio nazionale galileo (TNG) in Spain and with the very large telescope (VLT)-ESO in Chile. Observations were performed in several observing runs from 2003 to 2005. The observations at the TNG were obtained with the near infrared camera spectrograph (NICS) mostly with the Amici prism to obtain spectra in the range $0.9-2.3 \mu \mathrm{m}$ at $R \sim 75$. This low-resolution mode is excellent for investigating the QSO continuum shape, but also for detecting broad emission lines. Some QSOs were observed again with the IJ grism to obtain $0.9-1.45 \mu \mathrm{m}$ spectra at $R \sim 500$. Typical integration times ranged from $\sim 20 \mathrm{mn}$ to $\sim 3 \mathrm{~h}$. The observing strategy and data reduction are similar to those discussed in Maiolino et al. (2004).

The spectroscopic observations at ESO-VLT were done with the FORS2, along with the grism GRIS150I, to observe the range 6000-11000 Åat $R \sim 300$. These observations are mostly used to cover the short-wavelength part of some of the quasar spectra not properly sampled by the near-IR observations, but we also specifically observed a few quasars with no near-IR data with the specific aim of measuring the (SiIV+OIV)/CIV ratio. The total exposure times range from 30 to $60 \mathrm{mn}$. For some of the $z<5$ quasars observed with NICS, for which no FORS2 observations were available, we combine our near-IR spectra with optical data taken from Anderson et al. (2001).

\section{Results}

While individual spectra will be shown in a forthcoming paper (Gallerani et al., in prep.), in this letter we report the measurements of the (SiIV+OIV)/CIV ratio. To measure emission line fluxes, these low resolution quasar spectra were fitted with a model consisting of a power-law continuum and Gaussian profiles to model the emission lines. The power-law continuum was fitted using small spectral regions free of emission features

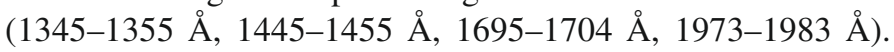
The SiIV+OIV] blend is unresolved and is fitted with a single Gaussian. Eight of our sources are broad absorption line (BAL) quasars. In these cases the measurement of the emission line flux is more complex and uncertain because of the absorption troughs, which are avoided when fitting both the continuum and the emission lines. Table 1 lists the (SiIV+OIV)/CIV ratio for all quasars in our sample, along with their redshift and continuum luminosity ( $\lambda L_{\lambda}$ at $1450 \AA$ ). Figure 1 (top panel) shows the measured (SiIV+OIV)/CIV ratios as a function of redshift. According to the calibration given in Nagao et al. (2006a), the observed ratios correspond to metallicities that are several times solar. No correlation is observed between the emission line ratio (hence metallicity) and redshift. Figure 2 shows the average spectra in the redshift intervals $4.0<z<5.5$ and $5.5<$ $z<6.4$ (obtained by first normalizing individual spectra to the
Table 1. (SiIV+OIV)/CIV measurements.

\begin{tabular}{|c|c|c|c|}
\hline Name & $z$ & $(\mathrm{SiIV}+\mathrm{OIV}) / \mathrm{CIV}$ & $\log \lambda L_{\lambda}^{a}$ \\
\hline SDSS J000239.4+255035 & 5.80 & $0.61 \pm 0.12$ & 46.93 \\
\hline SDSS J000552.3-000656 & 5.85 & $0.34 \pm 0.06$ & 46.20 \\
\hline SDSS J001714.66-100055. $4^{b}$ & 5.01 & $0.58 \pm 0.07$ & 46.56 \\
\hline SDSS J012004.82+141108. $2^{b}$ & 4.73 & $0.32 \pm 0.03$ & 46.09 \\
\hline SDSS J015642.11+141944. $3^{b}$ & 4.32 & $0.16 \pm 0.03$ & 46.62 \\
\hline SDSS J023137.6-072855 & 5.41 & $0.29 \pm 0.07$ & 46.56 \\
\hline SDSS J023923.47-081005. $1^{b}$ & 4.02 & $0.23 \pm 0.04$ & 46.58 \\
\hline SDSS J033829.3+002156 & 5.00 & $0.97 \pm 0.29$ & 46.46 \\
\hline SDSS J075618.1+410408 & 5.07 & $0.80 \pm 0.15$ & 46.53 \\
\hline SDSS J083643.8+005453 & 5.80 & $0.63 \pm 0.16$ & 46.97 \\
\hline SDSS J085210.89+535948. $9^{b}$ & 4.22 & $0.42 \pm 0.04$ & 46.53 \\
\hline SDSS J095707.67+061059.5 & 5.16 & $0.70 \pm 0.14$ & 46.65 \\
\hline SDSSp J102119.16-030937.2 & 4.70 & $0.35 \pm 0.12$ & 46.58 \\
\hline SDSS J103027.1+052455 & 6.28 & $0.59 \pm 0.20$ & 46.68 \\
\hline SDSS J104433.04-012502. $2^{b}$ & 5.78 & $0.40 \pm 0.13$ & 46.88 \\
\hline SDSS J104845.05+463718. $3^{b}$ & 6.20 & $0.42 \pm 0.20$ & 46.81 \\
\hline SDSS J114816.6+525150 & 6.40 & $0.41 \pm 0.08$ & 46.95 \\
\hline SDSS J120441.7-002150 & 5.05 & $0.57 \pm 0.12$ & 46.63 \\
\hline SDSSp J120823.8+001028 & 5.27 & $0.63 \pm 0.21$ & 46.07 \\
\hline SDSS J130608.2+035626 & 5.99 & $0.38 \pm 0.19$ & 47.32 \\
\hline SDSS J141111.3+121737 & 5.93 & $0.57 \pm 0.13$ & 46.58 \\
\hline SDSS J160254.2+422823 & 6.07 & $0.53 \pm 0.17$ & 46.90 \\
\hline SDSS J160320.89+072104.5 & 4.39 & $0.39 \pm 0.08$ & 46.85 \\
\hline SDSS J160501.21-011220.6 & 4.92 & $0.37 \pm 0.15$ & 46.46 \\
\hline SDSS J161425.13+464028.9 & 5.31 & $0.34 \pm 0.03$ & 46.62 \\
\hline SDSS J162331.8+311201 & 6.22 & $0.54 \pm 0.12$ & 46.54 \\
\hline SDSS J162626.50+275132.4 & 5.20 & $0.30 \pm 0.09$ & 46.94 \\
\hline SDSS J163033.9+401210 & 6.06 & $0.23 \pm 0.11$ & 46.38 \\
\hline SDSS J220008.7+001744 & 4.77 & $0.55 \pm 0.07$ & 46.58 \\
\hline SDSS J221644.0+001348 & 4.99 & $0.29 \pm 0.06$ & 46.18 \\
\hline
\end{tabular}

${ }^{a}$ Rest-frame continuum luminosity at $1450 \AA$, in units of $\mathrm{erg} \mathrm{s}^{-1} ;{ }^{b} \mathrm{BAL}$ quasars.

continuum at $\lambda 1450 \AA$, and avoiding BAL quasars), which are remarkably similar ${ }^{1}$, again highlighting any lack of evolution.

Our results can be compared with the line ratios and metallicities inferred at even lower redshifts, as obtained by Nagao et al. (2006a). It is important to use quasars with similar luminosities, since quasar metallicities show a strong correlation with luminosity. Given that flux limited surveys probe different luminosities at different redshifts, this may introduce an apparent redshift evolution. Therefore, from the $2<z<4$ templates in Nagao et al. (2006a), we select those in the highest luminosity bin $\left(-28.5>M_{\mathrm{B}}>-29.5\right)$, which match the quasar luminosities in our sample (which are also restricted to a relatively narrow range). In Fig. 2 the average spectra of $z>4$ quasars are compared with the average spectrum of luminous QSOs at $2.5<z<3.5$, which are all very similar. The lack of any evolution with redshift can be also appreciated in the bottom panel of Fig. 1, which shows the ratio (SiIV+OIV)/CIV measured in average quasar spectra at different redshifts (matched in luminosity).

\section{Discussion}

\subsection{The quasar metallicity "evolution"}

The apparent lack of evolution observed in Figs. 1-2 should not be interpreted as a lack of evolution of the BLR metallicity in individual AGNs. Indeed, Fig. 1 shows the average metallicity

\footnotetext{
1 Small differences in Ly $\alpha$ and NV are due to different absorption of the IGM and to different spectral resolution of the spectra.
} 

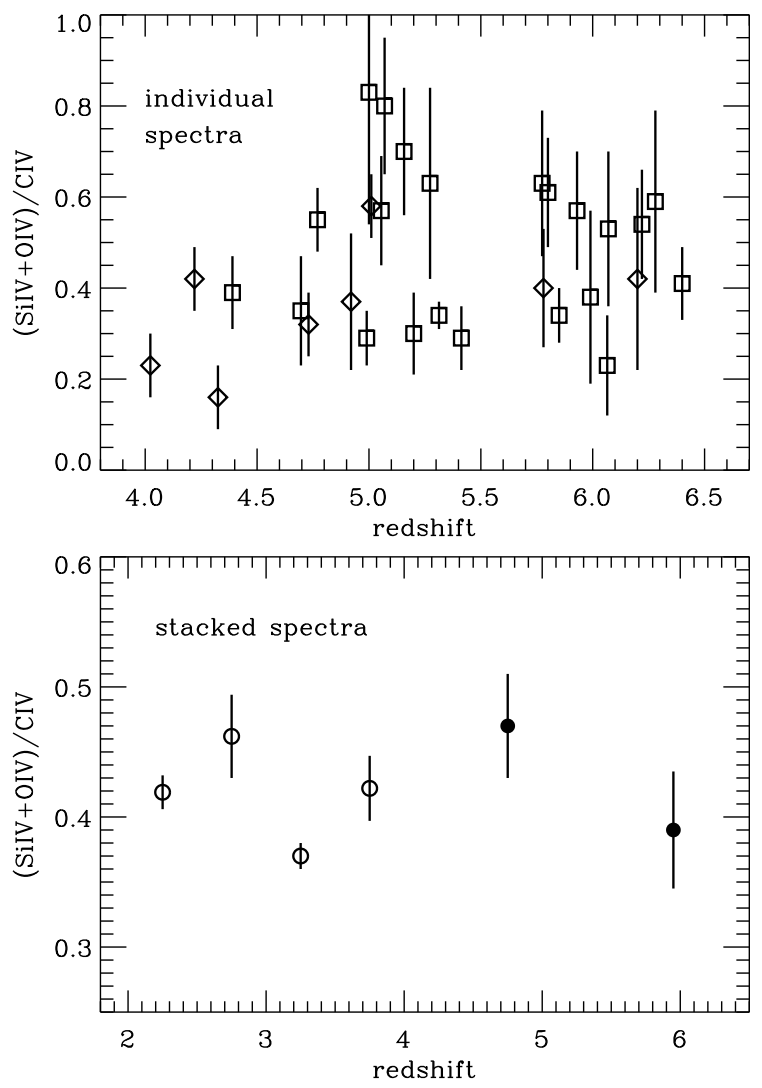

Fig. 1. (Upper) (SiIV+OIV)/CIV flux ratio as a function of redshift for quasars observed in this work. Squares indicate non-BAL quasars, while diamonds indicate BAL quasars. (Lower) (SiIV+OIV)/CIV ratio inferred from quasar stacked spectra, both for the high-redshift quasars presented here (filled symbols) and for the lower redshift quasars studied in Nagao et al. (2006a) (empty symbols).

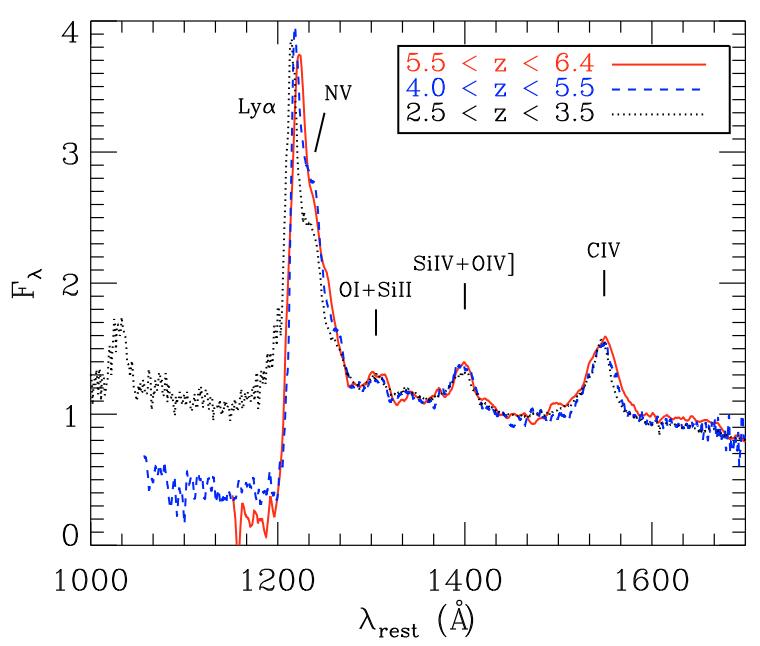

Fig. 2. Stacked spectra of quasars in different redshift bins (all normalized to $\lambda=1450 \AA$ ). The red solid line and the blue dashed line are the stacked spectra from our sample in the redshift intervals $5.5<z<6.4$ and $4.0<z<5.5$, respectively. The black dotted line is the SDSS quasars stacked spectrum in the redshift interval $2.5<z<3.5$.

of the BLR in quasars that are accreting at the given redshift, but does not trace the evolutionary path of individual quasars. The apparent lack of evolution in the BLR metallicity observed in Fig. 1 likely results from a combination of the BH-galaxy coevolution and selection effects. Indeed, to cross the detection threshold of the SDSS magnitude-limited survey, high-redshift quasars must have high luminosities, hence (even if accreting at the Eddington limit) high black hole masses. Most models predict that high black hole masses must have been accompanied by the formation of a massive host galaxy (e.g. Granato et al. 2004; Di Matteo et al. 2005; Hopkins et al. 2008; Li et al. 2007), which would result into the local $M_{\mathrm{BH}}-M_{\text {spheroid }}$ relationship. Therefore, by the time a quasar at any redshift is detectable in a magnitude-limited survey, its host galaxy must have evolved significantly and enriched its ISM significantly. The quasar feedback is another evolutionary effect that may yield to observational biases resulting in an apparent lack of metallicity evolution. Indeed, according to many models, during the early phases, when the host galaxy is still metal poor, the accreting black hole is embedded within the dusty ISM, and therefore difficult to detect in optical surveys. Only during the late evolutionary phases, when the galaxy is already metal rich, the quasar develops winds powerful enough to expel large quantities of gas and dust, so that the quasar becomes visible to optical observations.

To show these combined effects in a more quantitative way, we exploited the quasar-galaxy evolutionary models reported in Granato et al. (2004). In Fig. 3 we show the evolution of the gas metallicity in a massive galaxy forming in a dark halo of $M_{\text {halo }}=10^{13.2} M_{\odot}$, along with the evolution of the quasar bolometric luminosity and the total gas mass. While the host galaxy forms stars, the gas metallicity increases. At the same time the black hole accretion increases, yielding an increasingly high quasar luminosity. At $z>5$ the SDSS magnitude limit translates into a minimum quasar bolometric luminosity of about $10^{46} \mathrm{erg} \mathrm{s}^{-1}$ for detection. Figure 3 shows that by the time the quasar reaches such high luminosity (shaded region), the metallicity in the host galaxy is already higher than $\sim 3 Z_{\odot}$. At this time the model expects the quasar to be probably still embedded in gas and dust, although the quasar has already developed a wind expelling gas. It is not easy to identify the stage when the quasar becomes unobscured and detectable in optical surveys, since it depends on the detailed distribution of dust. We assume that the quasar becomes optically visible when more than half of the gas mass has been expelled. This is a somewhat arbitrary assumption, but we note that it is roughly consistent with what obtained by Lapi et al. (2006), who identify the epoch of unobscured accretion as starting a few $10^{7}$ yrs before the epoch of maximum luminosity. In Fig. 3 we note that by the time the quasar is unobscured (hatched region), the gas in the host galaxy has already reached a metallicity of about $\sim 4 Z_{\odot}$. Summarizing, the co-evolution of black holes and galaxies, combined with observational selection effects (mostly in optical surveys), naturally explains the finding that unobscured quasars of a given luminosity appear to have on average the same metallicity at any redshift.

\subsection{The extreme metallicities in the BLR}

According to Nagao et al. (2006a), the ratio (SiIV+OIV)/ CIV 0.4 observed in the stacked spectrum of the most distant quasars corresponds to a gas metallicity of $\sim 7 Z_{\odot}$. Such huge metallicities were also inferred by Nagao et al. (2006a) based on a much wider set of lines of lower redshift quasars. These high gas metallicities are not unrealistic. Indeed, the BLR is a small nuclear region (less than a few pc in the most luminous QSOs) with masses of a few times $\sim 10^{4} M_{\odot}$, which can be enriched in situ to super-solar metallicities within less than $10^{8} \mathrm{yrs}$ by having a supernova explosion less often than every $10^{4} \mathrm{yrs}$. More troublesome is that such high metallicities are not found in the stellar population of local massive galaxies, not even in their 


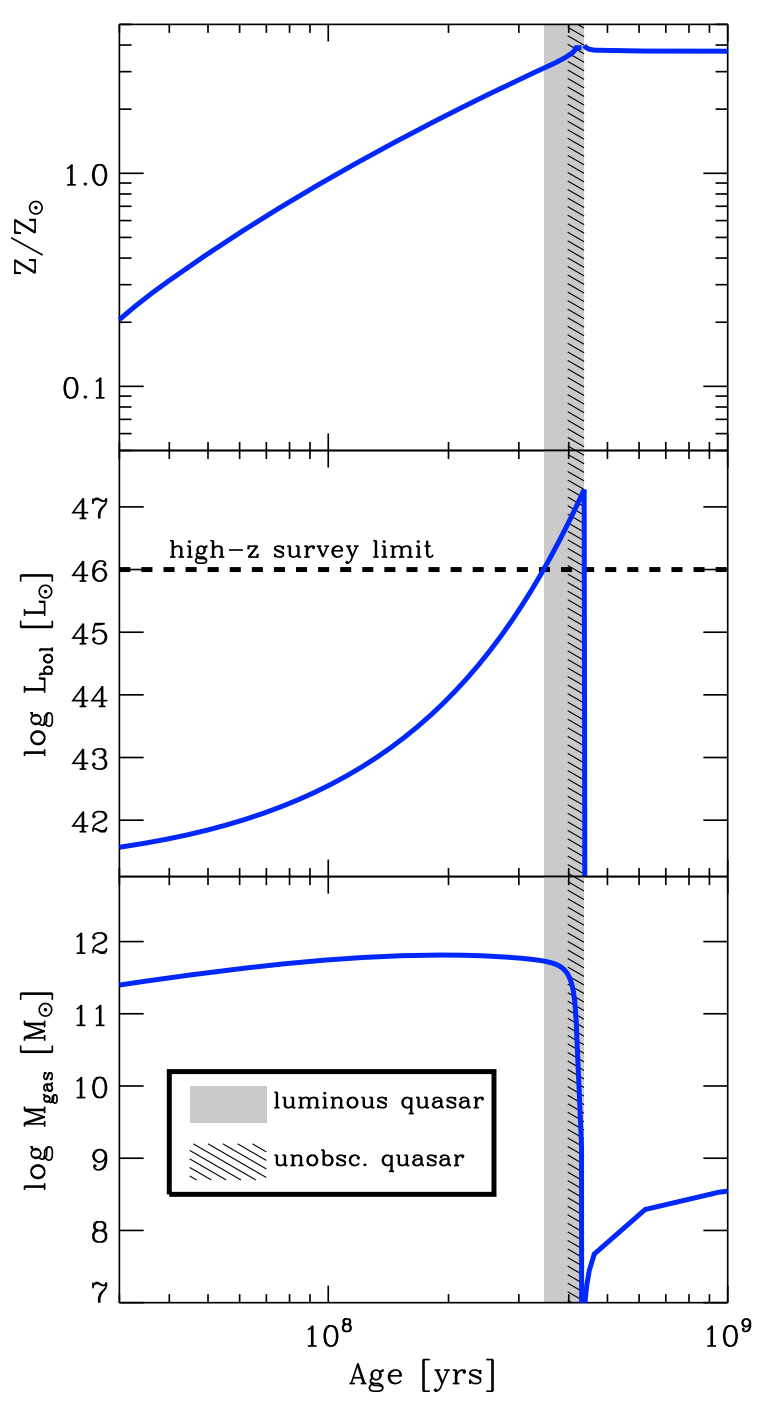

Fig. 3. Evolution of the gas metallicity (top), bolometric quasar luminosity (middle) and total gas mass (bottom) in the model by Granato et al. (2004). The horizontal dashed line shows the approximate minimum bolometric luminosity detectable at high- $z$ in the SDSS quasar survey. The shaded area shows the epoch when a high- $z$ quasar is luminous enough to be detected in the SDSS survey (if unobscured). The hatched area is the epoch when the quasar has ejected more than half of the gas mass, and it is likely unobscured and therefore detectable in optical surveys. By the time the quasar has reached the luminous, "unobscured" epoch the gas is already highly enriched.

central regions. Therefore, the high-metallicity gas observed in the BLR of high- $z$ quasars must either be expelled (as in quasar feedback models) or diluted by lower metallicity gas in the host galaxy before forming stars.

A related issue is how much the BLR metallicity is representative of the gas metallicity in the host galaxy. Investigating this issue would require accessing metallicity diagnostics that directly trace the quasar host galaxies. Host galaxies have been probed either through the narrow emission lines (only observable in obscured QSOs, e.g. Nagao et al. 2006b) or through the associated narrow absorption lines (e.g. D'Odorico et al. 2004), but only at intermediate redshifts $(z<3)$. It was found that the metallicity of the host galaxy is typically lower than observed in the BLR, but still super-solar, and again without any evidence of evolution with redshift. No direct measurements are currently available for the metallicity in the host galaxy of $z \sim 6$ quasars.
However, the detection of huge quantities of dust (Beelen et al. 2006) suggests that even the host of these quasars are heavily enriched.

\subsection{The carbon problem}

Relative elemental abundances are much more difficult to infer. However, the observational result that the ratio (SiIV+OIV)/CIV does not evolve suggests that the abundance of carbon relative to silicon and oxygen also does not evolve significantly. Large carbon abundances in the hosts of the most distant quasars is also suggested by the detection of strong [CII]158 $\mu \mathrm{m}$ and CO lines in the most distant quasars (Maiolino et al. 2005; Bertoldi et al. 2003). Carbon is mostly produced by AGB stars and planetary nebulae, most of which evolve on long timescales. Although the first AGB stars appear as soon as $\sim 50 \mathrm{Myr}$ after the onset of star formation, the bulk of carbon production occurs after about 1 Gyr, yielding a delayed enrichment with respect to $\alpha$-elements (e.g. oxygen) that are promptly produced by $\mathrm{SNII}^{2}$. At $z>6$ the age of the universe is less than $1 \mathrm{Gyr}$, hence stellar evolution may fall short of the time needed to produce the observed large carbon abundance. We note that this issue is independent of the size and mass of the BLR, making it just a pure timescale problem. Tackling this issue requires a more accurate determination of the carbon abundance, which may come from future high spectral resolution optical/near-IR observations or from future submm observations of far-IR fine structure lines (Maiolino 2008).

Acknowledgements. We are grateful to L. Silva and G. L. Granato for providing the electronic form of their models and for useful comments. This work was partially supported by the CONACyT project \#45948, by CONACyT grant for Ph. D. studies \#157846, by INAF and by ASI through contract ASIINAF I/016/07/0.

\section{References}

Anderson, S. F., Fan, X., \& Richards, G. T. 2001, AJ, 122, 503 Beelen, A., Cox, P., Benford, D. J., et al. 2006, ApJ, 642, 694 Bertoldi, F., Cox, P., Neri, R., et al. 2003, A\&A, 409, L47 Bresolin, F., Garnett, D. R., \& Kennicutt, R. C., Jr. 2004, ApJ, 615, 228 Di Matteo, T., Springel, V., \& Hernquist, L. 2005, Nature, 433, 604 D'Odorico, V., Cristiani, S., Romano, D. et al. 2004, MNRAS, 351, 976 Ferland, G. J., Baldwin, J. A., Korista, K. T., et al. 1996, ApJ, 461, 683 Freudling, W., Corbin, M. R., \& Korista, K. T. 2003, ApJ, 587, L67 Granato, G. L., De Zotti, G., Silva, L., et al. 2004, ApJ, 600, 580 Lapi, A., Shankar, F., Mao, J., et al. 2006, ApJ, 650, 42

Li, Y., Hernquist, L., \& Robertson, B. 2007, ApJ, 665, 187 Iwamuro, F., Kimura, M., Eto, S., et al. 2004, ApJ, 614, 69 Jiang, L., Fan, X., Vestergaard, M., et al. 2007, AJ, 134, 1150 Jiang, L., Fan, X., \& Vestergaard, M. 2008, ApJ, 679, 962 Kurk, J. D., Walter, F., Fan, X., et al. 2007, ApJ, 669, 32

Hamann, F. H., F., Warner, C., Dietrich, M., et al. 2007, ASP Conf. Ser., 373, 653

Hopkins, P. F., Hernquist, L., Cox, T. J., \& Kereš, D. 2008, ApJS, 175, 356 Maiolino, R. 2008, New Astron. Rev., 52, 339

Maiolino, R., Juarez, Y., Mujica, R., Nagar, N. M., \& Oliva, E. 2003, ApJ, 596, L155

Maiolino, R., Oliva, E., Ghinassi, F., et al. 2004, A\&A, 420, 889 Maiolino, R., Cox, P., Caselli, P., et al. 2005, A\&A, 440, L51 Nagao, T., Marconi, A., \& Maiolino, R. 2006a, A\&A, 447, 157 Nagao, T., Maiolino, R., \& Marconi, A. 2006b, A\&A, 447, 863 Pentericci, L., Fan, X., Rix, H.-W., et al. 2002, AJ, 123, 2151 Shemmer, O., Netzer, H., Maiolino, R., et al. 2004, ApJ, 614, 547 Warner, C., Hamann, F., \& Dietrich, M. 2004, ApJ, 608, 136

2 Silicon is also produced by SNIa and therefore is also probably en-
riched with a delay. However, Nagao et al. (2006a) find in their higher
resolution spectra that probably most of the emission in the SiIV+OIV blend is mostly due to OIV (see Tables 3-7). 